\title{
Profile of obinutuzumab for the treatment of patients with previously untreated chronic lymphocytic leukemia
}

This article was published in the following Dove Press journal:

OncoTargets and Therapy

3I August 2015

Number of times this article has been viewed

\section{Brian T Hill \\ Matt Kalaycio}

Department of Hematology and Medical Oncology, Taussig Cancer Institute, Cleveland Clinic, Cleveland, $\mathrm{OH}$, USA
Correspondence: Brian T Hill

Department of Hematologic Oncology and Blood Disorders, Taussig Cancer Institute, Cleveland Clinic, 9500 Euclid Avenue R35, Cleveland, OH 44I95, USA

$\mathrm{Tel}+$ I 216445945 I

Fax + I 2164449464

Email hillb2@ccf.org
Abstract: Chronic lymphocytic leukemia (CLL) is a hematologic malignancy derived from a clonal population of mature B-lymphocytes characterized by relatively low CD20 antigen expression. Although the disease often takes an indolent course, the majority of patients will eventually require therapy. Standard treatment for medically fit patients includes purine analogs and/or alkylating agents in addition to the type I anti-CD20 monoclonal antibody, rituximab. This therapy is inherently myelosuppressive and can result in significant morbidity and even mortality in patients with impaired performance status due to age and/or medical comorbidities. Historically, treatment options for the elderly or frail patient population were limited to monotherapy with the oral alkylating agent, chlorambucil, rituximab, or another type I anti-CD20 monoclonal antibody ofatumumab. Recently, a newer-generation anti-CD20 monoclonal antibody, obinutuzumab, was developed for patients with CLL. Obinutuzumab is a humanized type II monoclonal antibody that appears to have more direct antibody-dependent cell-mediated cytotoxicity (ADCC) and possibly more direct cytotoxicity in vitro than previously available type I antibodies. A large Phase III prospective randomized clinical trial for older patients with impaired renal function and/or significant medical comorbidities demonstrated that when compared to conventionally-dosed rituximab and chlorambucil, the combination of chlorambucil and obinutuzumab administered at a dose and schedule involving early loading doses improved response rates and progression-free survival without significantly increasing toxicity. Results of this pivotal trial led to the FDA (US Food and Drug Administration) approval of obinutuzumab in combination with chlorambucil for frontline treatment of CLL. Obinutuzumab expands the armamentarium of active and less-toxic targeted agents in the evolving treatment landscape of CLL, providing physicians and patients with an additional therapeutic option.

Keywords: obinutuzumab, monoclonal antibody, CLL, chlorambucil, cell-mediated cytotoxicity, combination regimens

\section{Historical perspective: conventional chemotherapy}

Chronic lymphocytic leukemia (CLL) is diagnosed in more than 15,000 new patients annually in the United States. Median age at diagnosis is 73 years. ${ }^{1,2}$ Although many patients have an indolent course and may never require therapy, the majority of patients with CLL will eventually need treatment due to progressive bone marrow failure, compressive lymphadenopathy/organomegaly, and/or "B" symptoms including fevers, night sweats, or unintentional weight loss. Initial therapy for patients in need of treatment for CLL has historically utilized a purine nucleoside analog such as fludarabine or pentostatin and/or an alkylating agent such as chlorambucil or cyclophosphamide. Combining agents from both of these classes of drugs has additive clinical benefit: fludarabine and cyclophosphamide (FC) demonstrated improved response rates and 
incremental progression-free survival (PFS) improvement when compared to fludarabine monotherapy. ${ }^{3-5}$ Treatmentrelated adverse events are common with $\mathrm{FC}$, particularly grade $3 / 4$ hematologic toxicities and infection. FC was frequently used for medically fit patients with CLL, but overall survival (OS) of CLL patients was not improved relative to single-agent treatment. Of note, the patients with deletion of chromosome 11q appeared to benefit the most from the addition of the alkylating agent cyclophosphamide.

\section{Benefit to addition of monoclonal antibody to CLL treatment}

One of the most significant advances in combination regimens for up-front treatment of CLL in the past decade was the addition of the first monoclonal antibody against CD20, rituximab. Rituximab is a conventional type I monoclonal antibody. It elicits its antitumor effects through multiple mechanisms, including antibody-dependent cell-mediated cytotoxicity (ADCC), complement-mediated cytotoxicity (CMC), and possibly direct cytotoxicity by induction of apoptosis. ${ }^{6,7}$

In follow-up to the recognition of the strong additional clinical benefit of rituximab when added to chemotherapy for non-Hodgkin lymphoma (NHL), Phase I-II trials in CLL demonstrated clinical activity of rituximab in CLL alone or in combination with fludarabine. ${ }^{8,9}$ Given these encouraging results, rituximab was added to the fludarabine and cyclophosphamide chemotherapy backbone (FCR) for up-front treatment of medically fit CLL patients. The FCR combination utilizes rituximab at standard doses used in NHL $\left(375 \mathrm{mg} / \mathrm{m}^{2}\right)$ during the first cycle of therapy followed by an escalated dose of $500 \mathrm{mg} / \mathrm{m}^{2}$ for subsequent cycles. Initial study of the FCR regimen found that it produced a very high overall response rate (ORR) of $95 \% .{ }^{10}$ This led to a randomized Phase III trial through the German CLL Study Group (CLL 8) comparing FCR vs FC, which demonstrated improved ORR (90\% vs $80 \%$, $P<0.001$ ) and PFS (median, 51.8 months [95\% confidence interval (CI), 46.2-57.6] vs 32.8 months [95\% CI, 29.6-36.0]; $P<0.0001$ ) with FCR. ${ }^{11}$ A major conclusion of this study was that - for the first time - introduction of an additional agent to an existing treatment regimen for CLL resulted in an improvement in OS (3-year survival, $87 \%$ vs $83 \%$, respectively; hazard ratio [HR], 0.67 [95\% CI, $0.48-0.92] ; P=0.01$ ). Treatment was significantly less effective for the subset of patients with the high-risk cytogenetic abnormality in which the tumor suppressor gene $p 53$ is absent due to deletion of chromosome $17 \mathrm{p}$. Severe infections and grade $3 / 4$ myelosuppression were common, and treatment-related mortality was $>2 \%$, but comparable in the FCR and FC groups.
Subsequently, rituximab has been added to other CLL chemotherapy regimens, including bendamustine (BR), pentostatin, and others. ${ }^{12,13}$ More recently, a head-to-head prospective Phase III trial of FCR vs BR for medically fit patients with CLL in need of treatment was performed by the German CLL Study Group (CLL 13). ${ }^{14}$ Enrolled patients were devoid of major comorbidities and had normal renal function. Median age was 62 years. The ORR in both arms was $97.8 \%$. The complete response (CR) rate was $40.7 \%$ with FCR compared to $31.5 \%$ with $\mathrm{BR}(P=0.026)$. More patients treated with FCR achieved negative testing for minimal residual disease (MRD). Median PFS was 53.7 months for the FCR arm and 43.2 months for the BR arm (HR, 1.589 [95\% CI, 1.25-2.079]; $P=0.001)$. However, the PFS difference was not statistically significant for patients over the age of 65 or in patients with comorbidities, and OS was not significantly different between the two groups. Treatment-related mortality was $3.9 \%$ (FCR) and $2.1 \%$ (BR), respectively. These results have led different investigators to alternative conclusions regarding the optimal frontline therapy for CLL. While FCR may offer higher response rates, it is associated with more toxicity without an OS benefit, and the PFS for patients with advanced age or comorbidities is comparable to BR.

\section{Optimizing CD20-targeted monoclonal antibody}

Given the additive benefit of rituximab to chemotherapy regimens, there has been considerable interest in improving anti-CD20 monoclonal antibody technology for therapeutic benefit. In particular, rituximab may not be the optimal agent to target CLL cells, which are characterized by relatively low cell surface expression of CD20. The first so-called second-generation anti-CD20 monoclonal antibody was ofatumumab. Ofatumumab is a fully humanized anti-CD20 monoclonal antibody whose epitope is a small loop of the extracellular domain of CD20, distinct from the binding site for rituximab (Figure 1). ${ }^{6,15}$ Preclinical studies suggested that ofatumumab has higher CD20 avidity than rituximab, possibly leading to more $\mathrm{CMC} .{ }^{16}$

In the case of relapsed/refractory CLL, a large Phase II study of ofatumumab established this agent as having clinical activity in previously treated patients. ${ }^{17}$ Ofatumumab was administered as a lead-in flat dose of $300 \mathrm{mg}$ during the 1st week, followed by weekly doses of 2,000 $\mathrm{mg}$ for 7 doses during the first 2 months, and then monthly for an additional 4 doses. The ORR was $51 \%$ in the entire cohort, including those with bulky disease, and did not appear different in patients with or without prior rituximab exposure. Responses were almost 


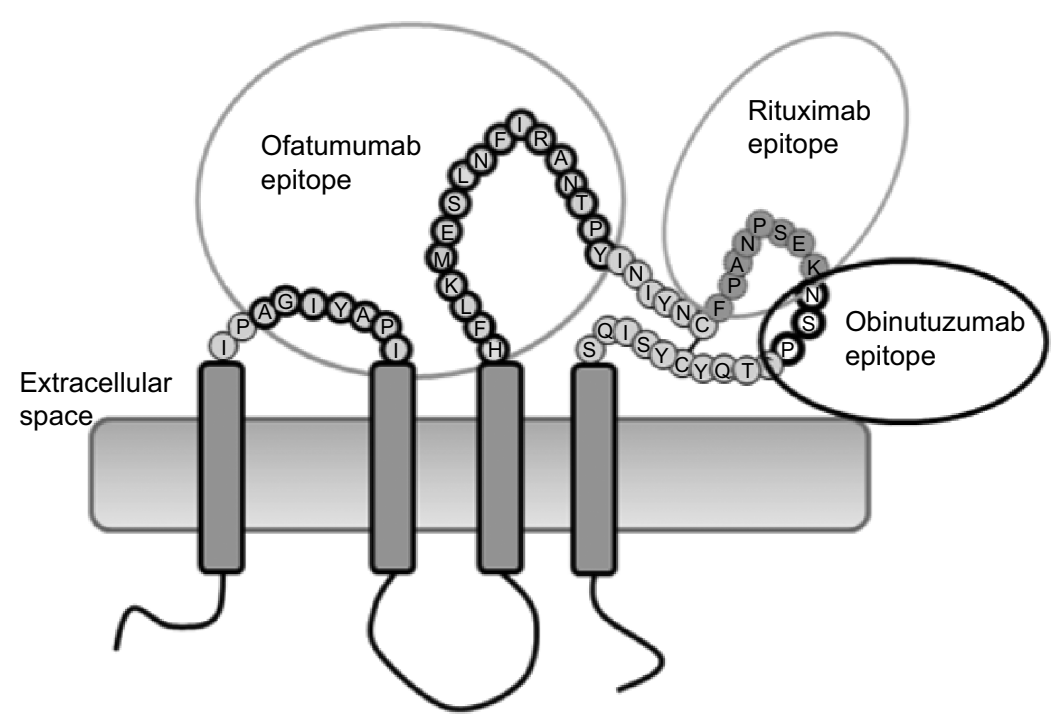

Figure I Structure of CD20 and epitope targets of ofatumumab, rituximab, and obinutuzumab (GAI0I).

Notes: The CD20 transmembrane receptor is shown with epitopes for binding of ofatumumab, rituximab, and obinutuzumab. Adapted with permission from Klein C, Lammens A, Schafer W, et al. Epitope interactions of monoclonal antibodies targeting CD20 and their relationship to functional properties. MAbs. 20I3;5(I):22-33. ${ }^{15}$

exclusively partial remissions with a single CR. The median duration of response was approximately 6 months.

\section{Obinutuzumab: first FDA-approved anti-CD20 type II monoclonal antibody}

In contrast to ofatumumab and rituximab, which are type I monoclonal antibodies targeted against CD20, obinutuzumab (formerly GA101) is a type II antibody. Type I antibodies are strong activators of complement. Preclinical evidence suggests that a large part of the cytotoxic effect of the type I antibodies is in fact due to CMC. In contrast, type II antibodies have minimal $\mathrm{CMC}$ but appear to have more direct cellular cytotoxicity. Both type I and type II antibodies have some level of ADCC. Rituximab induces caspase-dependent changes suggestive of induction of apoptosis in vitro, but it has not been convincingly established that this mechanism of action is relevant in vivo. ${ }^{6}$ Type II monoclonal antibodies induce cell death via release of cytotoxic contents of intracellular lysosomes in the target cells, bypassing the antiapoptotic Bcl-2 family of proteins that may be responsible for significant chemotherapy resistance. ${ }^{18}$ Obinutuzumab is a humanized monoclonal antibody that targets the same extracellular domain in the CD20 antigen as does rituximab but recognizes a nonoverlapping epitope (Figure 1). ${ }^{15}$ However, the Fc region of obinutuzumab was modified with additional carbohydrate groups ("glyco-engineered") to improve binding capacity to effector cells, including neutrophils, natural killer cells, thereby possibly enhancing ADCC. ${ }^{19,20}$
The activity of obinutuzumab was evaluated in a Phase I/II trial in patients with relapsed/refractory indolent NHL or aggressive NHL (diffuse large B-cell lymphoma and mantle cell lymphoma) most of whom were previously treated with rituximab. ${ }^{21,22}$ There appeared to be a dose-dependent response with ORR of 55\% for subjects with indolent NHL and $37 \%$ for patients with aggressive NHL receiving two loading doses of $1,600 \mathrm{mg}$, followed by doses of $800 \mathrm{mg}$ every 2 weeks. The therapy was well tolerated, with a high frequency of infusion-related reactions (IRRs) that were predominantly grade $1-2$. Premedication with acetaminophen and antihistamine was required, and glucocorticoid was given to high-risk patients. Serum concentrations of obinutuzumab were significantly higher, earlier in the course of therapy, for patients treated with loading doses rather than continuous dosing at $400 \mathrm{mg}$ every 2 weeks (Figure 2). This is particularly important, as it provided direct clinical evidence for the pharmacodynamic and pharmacokinetic models in which early doses of monoclonal antibody do not necessarily reach their target, as the initial drug is absorbed by both target cells and off-target immune effector cells.

\section{Less-toxic therapy for older/frail CLL patients: role of chlorambucil alone or in combination with monoclonal antibody}

For patients unfit for aggressive cytotoxic chemotherapy regimens, treatment for CLL has historically been considered palliative. For many years, oral chlorambucil monotherapy 


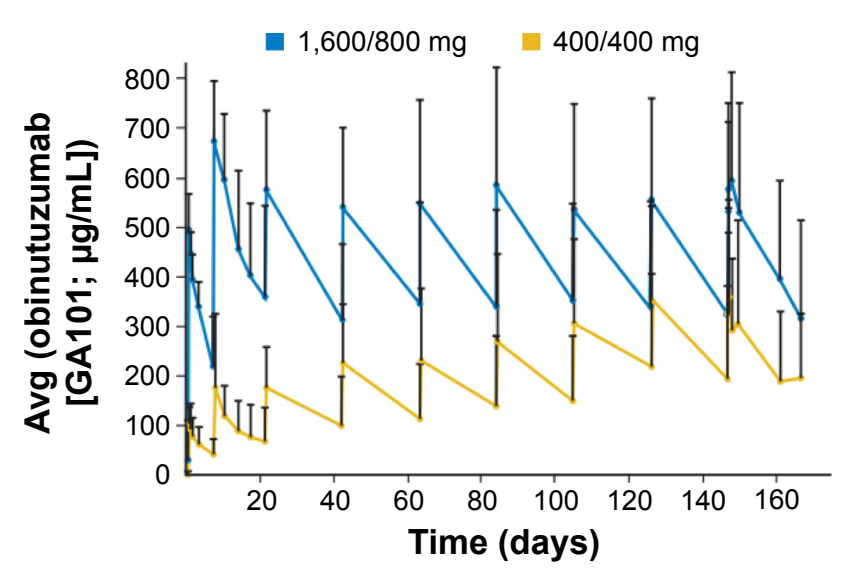

Figure 2 Pharmacokinetics of obinutuzumab.

Notes: Serum concentrations of obinutuzumab based on two loading doses of I,600 mg followed by doses of $800 \mathrm{mg}$ every 2 weeks (blue line) vs continuous dosing with $400 \mathrm{mg}$ every 2 weeks (yellow line), for patients with relapsed/ refractory NHL. Reprinted with permission. C2013 American Society of Clinical Oncology. all rights reserved. Morschhauser FA, Cartron G, Thieblemont C, et al. Obinutuzumab (GAIOI) monotherapy in relapsed/refractory diffuse large b-cell lymphoma or mantle-cell lymphoma: results from the phase II GAUGUIN study. J Clin Oncol. 2013;31(23):2912-2919. ${ }^{21}$

Abbreviation: NHL, non-Hodgkin lymphoma.

was often used as an inexpensive and minimally toxic regimen. Previous prospective studies in the frontline setting demonstrated that when used alone, chlorambucil has an ORR of approximately $30 \%-50 \%$, with very few complete remissions, and PFS $<2$ years. ${ }^{5,23-26}$ The dose and schedule of the administration of chlorambucil are variable and have not been subjected to intense optimization strategies.

Because addition of monoclonal antibody provides significant additional therapeutic benefit to most chemotherapeutic agents for B-cell malignancies, Phase II trials were conducted to test the combination of chlorambucil with rituximab. ${ }^{27,28}$ This treatment strategy was designed for older or frail CLL patients in whom intensive chemoimmunotherapy was contraindicated due to potential toxicity. ORR in these trials was $>80 \%$, with PFS of approximately 2 years or longer with the addition of rituximab maintenance. ${ }^{28}$

In addition, a Phase III trial for the first-line treatment of CLL patients compared ofatumumab in combination with chlorambucil to chlorambucil alone. ${ }^{29}$ For patients with a median age of 69, the majority of whom had comorbidities, PFS was significantly longer in the patients treated with ofatumumab and chlorambucil relative to chlorambucil alone (22.4 months vs 13.1 months, $P<0.001$ ). ORR was $82 \%$ vs $69 \%, P=0.001$, favoring the combination therapy group, and CR rates were also higher ( $12 \%$ vs $1 \%$ ). MRD testing in the peripheral blood or bone marrow was negative in 37\% patients treated with the combination therapy. Toxicities included IRRs and neutropenia, but the incidence of grade 3/4 infection was low and not different between the two groups.
Subsequent to the presentation of these data, ofatumumab gained a new indication from the FDA (US Food and Drug Administration) for frontline treatment of CLL.

\section{Obinutuzumab for previously untreated CLL}

The clinical efficacy of the single-agent obinutuzumab was tested at two different dose levels in previously untreated CLL in a randomized Phase II study. ${ }^{30}$ Obinutuzumab was given at either 1,000 mg on days 1 and 2 (dose was split into $100 \mathrm{mg}$ on day 1 and $900 \mathrm{mg}$ on day 2 to mitigate IRRs), then $1,000 \mathrm{mg}$ on days 8 and 15 of cycle 1 and every 21 days for cycles $2-8$ thereafter, or at 2,000 $\mathrm{mg}$ on days $1-3$ (100 $\mathrm{mg}$ on day 1 , $900 \mathrm{mg}$ on day $2,1,000 \mathrm{mg}$ on day 3 ), then on days 8 and 15 of cycle 1 , followed by once every 21 days for cycles $2-6$. The ORR was $67 \%$ in the higher-dose group vs $49 \%$ in the lower-dose group $(P=0.08)$, in this 80 -patient study. CR was achieved in 8 out of 39 patients in the high-dose group (21\%) and 2 out of 41 patients (5\%) in the low-dose group.

The German CLL Study Group studied the efficacy of obinutuzumab in combination with chlorambucil in a pivotal Phase III three-arm prospective clinical trial for previously untreated CLL patients in need of therapy. ${ }^{31}$ Importantly, inclusion criteria were designed to specifically enroll patients who were not candidates for more intensive therapy. Thus, the Cumulative Illness Rating Scale was used, which quantifies comorbidities and functional status to provide objective assessment of subjects' burden of medical conditions. ${ }^{32}$ This score ranges from 0 to 56 , with high scores indicating poorer overall health status. To qualify for inclusion in the study, patients were required to have a score of higher than 6 or to have a creatinine clearance less than $70 \mathrm{~mL} /$ minute (but $\geq 30$ ). Patients were randomly assigned to either chlorambucil monotherapy or chlorambucil in combination with either rituximab or obinutuzumab. The dose and schedule of administration are shown in Figure 3. The study enrolled 281 patients. The median age was 73 years, which is the median age of patients at the time of diagnosis of CLL. The significant majority of patients $(82 \%)$ had three or more comorbid conditions.

Although there was a higher incidence of grade $3 / 4$ neutropenia in the patients treated with obinutuzumab and chlorambucil, this did not translate into a significant difference in infection rates, which were similar between the three treatment groups. The rate of grade 3/4 IRR was higher in the obinutuzumab and chlorambucil arm relative to the rituximab and chlorambucil arm ( $20 \%$ vs $4 \%$, respectively). Interestingly, IRRs beyond the initial infusion were relatively 


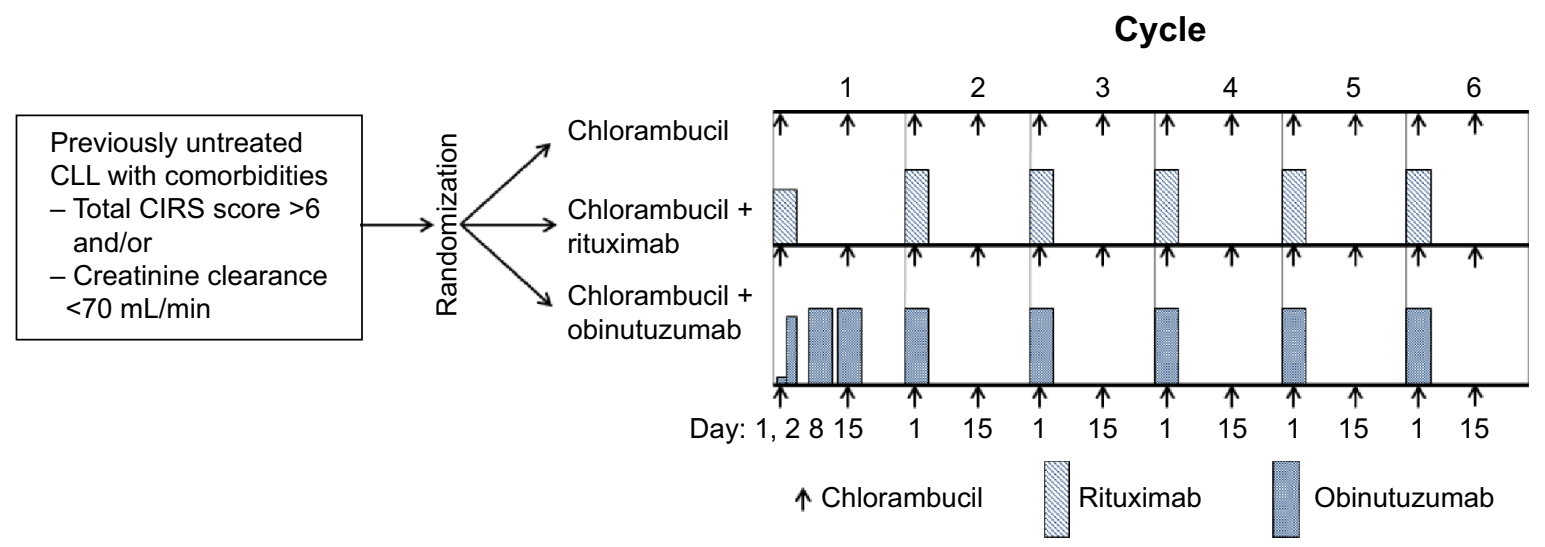

Figure 3 Schematic representation of pivotal Phase III German CLLI 3 trial.

Notes: Patients were enrolled and randomly assigned on a 1:2:2 ratio to chlorambucil alone, rituximab plus chlorambucil, or obinutuzumab plus chlorambucil on 28-day cycles. Chlorambucil was dosed at $0.5 \mathrm{mg} / \mathrm{kg}$ on days I and I . Rituximab was administered intravenously at a dose of $375 \mathrm{mg} / \mathrm{m}^{2}$ on day I of cycle I and $500 \mathrm{mg} / \mathrm{m}^{2}$ on day I of cycles 2-6. Obinutuzumab was administered intravenously at a dose of I,000 mg on day I initially and - during subsequent amendments - over a period of 2 days, days 8 and 15 of cycle I, and on day I of cycles $2-6 .^{31}$

Abbreviations: CLL, chronic lymphocytic leukemia; CIRS, Cumulative Illness Rating Scale.

uncommon in obinutuzumab-treated patients, relative to rituximab. There were 15 cases of tumor lysis syndrome ( 6 cases were grade $\geq 3$ ); all except one occurred in patients treated with chlorambucil and obinutuzumab. The median number or cycles completed was 6 in each arm. The median cumulative dose of chlorambucil was comparable in the three treatment arms.

The ORR was higher in the obinutuzumab + chlorambucil arm relative to the rituximab + chlorambucil arm $(77.7 \%$ vs $65.1 \%, P<0.001)$, with higher rates of CR $(20.7 \%$ vs $7.0 \%)$ favoring obinutuzumab + chlorambucil. MRD negativity in bone marrow was significantly higher in those patients treated with obinutuzumab and chlorambucil compared to rituximab and chlorambucil (19.5\% vs $2.6 \%$ ).

These response rates translated into a PFS benefit for obinutuzumab and chlorambucil as compared with chlorambucil alone (median PFS, 26.7 months vs 11.1 months). The HR for progression or death was 0.18 (95\% CI, 0.13-0.24; $P<0.001)$. The median PFS of 26.7 months in the group treated with obinutuzumab and chlorambucil was statistically significantly longer than that in the group treated with rituximab and chlorambucil (15.2 months, HR, 0.39 [95\% CI, 0.31-0.49]). The PFS benefit of obinutuzumab and chlorambucil did translate into an OS benefit relative to chlorambucil, but not the combination of rituximab and chlorambucil.

\section{Perspective}

Therapy for CLL has advanced significantly over recent decades but has tended to build on more aggressive chemoimmunotherapy regimens. This has culminated with the advent of FCR, which produces nearly $100 \%$ response rates when used in first-line treatment of patients without $17 \mathrm{p}$ deletion. PFS is long with FCR - over 4 years - but the cost of this high level of efficacy is significant toxicity, with treatmentrelated mortality exceeding $4 \%$. Although the benefit of FCR in terms of response rates and PFS relative to BR is observed for young, fit patients, there is no OS benefit and the PFS and ORR advantage is not maintained for patients older than 65 years or with comorbidities. In addition, there is a significant risk of protracted myelosuppression and secondary malignancies, including myelodysplastic syndrome and treatment-related acute leukemia. In total, given that the median age of CLL is 73 years at the time of diagnosis, and there is a significant lag time between diagnosis and indication for therapy, it is fair to say that the majority of CLL patients in need of treatment are not good candidates for FCR. Although BR is less toxic, it is highly myelosuppressive and, therefore, still difficult to deliver in older patients. Given the additional burdens of comorbid conditions with advanced age, a highly effective regimen for the frail CLL patient population has been an unmet medical need for many years.

There now exists level I evidence that all of the monoclonal antibodies (rituximab, ofatumumab, and obinutuzumab) significantly improve ORR and PFS when added to minimally myelosuppressive doses of chlorambucil. ${ }^{29,31}$ Furthermore, in head-to-head comparison, the obinutuzumab and chlorambucil regimen outperforms the rituximab and chlorambucil regimen. ${ }^{31}$ It is noteworthy that the regimen utilized of obinutuzumab and chlorambucil three loading doses (with the first dose split over days 1 and 2) during the first cycle of therapy, whereas the protocol for rituximab + chlorambucil relied on a more traditional 
"step-up" dosing of rituximab, in which the antibody was given at $375 \mathrm{mg} / \mathrm{m}^{2}$ on day 1 of the first cycle, followed by $500 \mathrm{mg} / \mathrm{m}^{2}$ on day 1 of cycles $2-6$. Given the known pharmacokinetics of monoclonal antibodies with high clearance early in the course of treatment, this dosing schedule clearly favored obinutuzumab, conferring a higher ORR and CR rate when administered at higher doses earlier. ${ }^{30}$ Therefore, one must be cautious in concluding that obinutuzumab is truly a superior monoclonal antibody than rituximab. In fact, when compared to the outcomes of treatment with ofatumumab and chlorambucil combination, those with the obinutuzumab and chlorambucil combination appear similar (ORR, 82\% vs 78.4\%; PFS, 22.4 vs 26.7 months, respectively). ${ }^{29,31}$ Therefore, the most accurate statement based on available data is that when combined with chlorambucil, obinutuzumab given at the dose and schedule of the German CLL11 trial produced superior response rates and PFS relative to chlorambucil in combination with conventionally-dosed rituximab.

It is premature to extrapolate from this experience to conclude that obinutuzumab should replace rituximab with intensive chemotherapeutic regimens (FC or BR). In particular, the early experience with these combinations has been noteworthy for relatively high rates of grade $3 / 4$ hematologic toxicity requiring treatment delays or discontinuations. ${ }^{33}$ Clearly, more safety and efficacy data are needed with more intensive chemotherapy regimens in the frontline setting.

With the B-cell receptor pathway inhibitors ibrutinib and idelalisib now available, and other highly active agents in development, including the very promising Bcl-2 inhibitor, Venetoclax, the tides are shifting in the management of CLL toward more targeted, less-toxic therapies. For now, these drugs are available only at the time of relapse or, in the relatively uncommon case of $17 \mathrm{p}$ deletion, at the time of diagnosis. The availability of obinutuzumab for the frontline treatment of CLL is a welcome addition to the treatment landscape and is particularly appealing for the older/frailer patient for whom intensive therapy is not a viable option.

\section{Disclosure}

The authors report no conflicts of interest in this work.

\section{References}

1. Howlader N, Noone AM, Krapcho M, et al. SEER Cancer Statistics Review, 1975-2010. Bethesda, MD: National Cancer Institute; 2012.

2. Slager SL, Benavente Y, Blair A, et al. Medical history, lifestyle, family history, and occupational risk factors for chronic lymphocytic leukemia/ small lymphocytic lymphoma: the InterLymph Non-Hodgkin Lymphoma Subtypes Project. J Natl Cancer Inst Monogr. 2014;2014(48):41-51.
3. Flinn IW, Neuberg DS, Grever MR, et al. Phase III trial of fludarabine plus cyclophosphamide compared with fludarabine for patients with previously untreated chronic lymphocytic leukemia: US Intergroup Trial E2997. J Clin Oncol. 2007;25(7):793-798.

4. Eichhorst BF, Busch R, Hopfinger G, et al. Fludarabine plus cyclophosphamide versus fludarabine alone in first-line therapy of younger patients with chronic lymphocytic leukemia. Blood. 2006;107(3): 885-891.

5. Catovsky D, Richards S, Matutes E, et al. Assessment of fludarabine plus cyclophosphamide for patients with chronic lymphocytic leukaemia (the LRF CLL4 Trial): a randomised controlled trial. Lancet. 2007; 370(9583):230-239.

6. Beers SA, Chan CH, French RR, Cragg MS, Glennie MJ. CD20 as a target for therapeutic type I and II monoclonal antibodies. Semin Hematol. 2010;47(2):107-114.

7. Dalle S, Dumontet C. [Rituximab: mechanism of action and resistance]. Bull Cancer. 2007;94(2):198-202. French.

8. Byrd JC, Murphy T, Howard RS, et al. Rituximab using a thrice weekly dosing schedule in B-cell chronic lymphocytic leukemia and small lymphocytic lymphoma demonstrates clinical activity and acceptable toxicity. J Clin Oncol. 2001;19(8):2153-2164.

9. Byrd JC, Peterson BL, Morrison VA, et al. Randomized phase 2 study of fludarabine with concurrent versus sequential treatment with rituximab in symptomatic, untreated patients with B-cell chronic lymphocytic leukemia: results from Cancer and Leukemia Group B 9712 (CALGB 9712). Blood. 2003;101(1):6-14.

10. Keating MJ, O'Brien S, Albitar M, et al. Early results of a chemoimmunotherapy regimen of fludarabine, cyclophosphamide, and rituximab as initial therapy for chronic lymphocytic leukemia. J Clin Oncol. 2005; 23(18):4079-4088.

11. Hallek M, Fischer K, Fingerle-Rowson G, et al. Addition of rituximab to fludarabine and cyclophosphamide in patients with chronic lymphocytic leukaemia: a randomised, open-label, phase 3 trial. Lancet. 2010; 376(9747):1164-1174.

12. Fischer K, Cramer P, Busch R, et al. Bendamustine in combination with rituximab for previously untreated patients with chronic lymphocytic leukemia: a multicenter phase II trial of the German Chronic Lymphocytic Leukemia Study Group. J Clin Oncol. 2012;30(26):3209-3216.

13. Kay NE, Geyer SM, Call TG, et al. Combination chemoimmunotherapy with pentostatin, cyclophosphamide, and rituximab shows significant clinical activity with low accompanying toxicity in previously untreated B chronic lymphocytic leukemia. Blood. 2007;109(2):405-411.

14. Eichhorst B, Fink AM, Busch R, et al. Frontline chemoimmunotherapy with fludarabine $(\mathrm{F})$, cyclophosphamide $(\mathrm{C})$, and rituximab (R) (FCR) shows superior efficacy in comparison to bendamustine (B) and rituximab (BR) in previously untreated and physically fit patients (pts) with advanced chronic lymphocytic leukemia (CLL): final analysis of an international, randomized study of the German CLL Study Group (GCLLSG) (CLL10 Study). Poster presented at: American Society of Hematology Annual Meeting, 2014, Washington, DC.

15. Klein C, Lammens A, Schafer W, et al. Epitope interactions of monoclonal antibodies targeting CD20 and their relationship to functional properties. MAbs. 2013;5(1):22-33.

16. Teeling JL, French RR, Cragg MS, et al. Characterization of new human CD20 monoclonal antibodies with potent cytolytic activity against non-Hodgkin lymphomas. Blood. 2004;104(6):1793-1800.

17. Wierda WG, Kipps TJ, Mayer J, et al. Ofatumumab as single-agent CD20 immunotherapy in fludarabine-refractory chronic lymphocytic leukemia. J Clin Oncol. 2010;28(10):1749-1755.

18. Ivanov A, Beers SA, Walshe CA, et al. Monoclonal antibodies directed to CD20 and HLA-DR can elicit homotypic adhesion followed by lysosome-mediated cell death in human lymphoma and leukemia cells. J Clin Invest. 2009;119(8):2143-2159.

19. Golay J, Da Roit F, Bologna L, et al. Glycoengineered CD20 antibody obinutuzumab activates neutrophils and mediates phagocytosis through CD16B more efficiently than rituximab. Blood. 2013;122(20): 3482-3491. 
20. Mossner E, Brunker P, Moser S, et al. Increasing the efficacy of CD20 antibody therapy through the engineering of a new type II anti-CD20 antibody with enhanced direct and immune effector cell-mediated B-cell cytotoxicity. Blood. 2010;115(22):4393-4402.

21. Morschhauser FA, Cartron G, Thieblemont C, et al. Obinutuzumab (GA101) monotherapy in relapsed/refractory diffuse large b-cell lymphoma or mantle-cell lymphoma: results from the phase II GAUGUIN study. J Clin Oncol. 2013;31(23):2912-2919.

22. Salles GA, Morschhauser F, Solal-Celigny P, et al. Obinutuzumab (GA101) in patients with relapsed/refractory indolent non-Hodgkin lymphoma: results from the phase II GAUGUIN study. J Clin Oncol. 2013; 31(23):2920-2926.

23. Eichhorst BF, Busch R, Stilgenbauer S, et al. First-line therapy with fludarabine compared with chlorambucil does not result in a major benefit for elderly patients with advanced chronic lymphocytic leukemia. Blood. 2009;114(16):3382-3391.

24. Rai KR, Peterson BL, Appelbaum FR, et al. Fludarabine compared with chlorambucil as primary therapy for chronic lymphocytic leukemia. N Engl J Med. 2000;343(24):1750-1757.

25. Hillmen P, Skotnicki AB, Robak T, et al. Alemtuzumab compared with chlorambucil as first-line therapy for chronic lymphocytic leukemia. J Clin Oncol. 2007;25(35):5616-5623.

26. Knauf WU, Lissichkov T, Aldaoud A, et al. Phase III randomized study of bendamustine compared with chlorambucil in previously untreated patients with chronic lymphocytic leukemia. J Clin Oncol. 2009;27(26): 4378-4384.

27. Hillmen P, Gribben JG, Follows GA, et al. Rituximab plus chlorambucil as first-line treatment for chronic lymphocytic leukemia: final analysis of an open-label phase II study. J Clin Oncol. 2014;32(12):1236-1241.
28. Foa R, Del Giudice I, Cuneo A, et al. Chlorambucil plus rituximab with or without maintenance rituximab as first-line treatment for elderly chronic lymphocytic leukemia patients. Am J Hematol. 2014;89(5): $480-486$.

29. Hillmen P, Robak T, Janssens A, Govindbabu K. Ofatumumab + chlorambucil versus chlorambucil alone in patients with untreated chronic lymphocytic leukemia (CLL): results of the phase III Study Complement 1. Poster presented at: American Society of Hematology Annual Meeting, 2013, Washington, DC.

30. Flynn JM, Byrd JC, Kipps TJ, Boxer M. Obinsutuzumab (GA101) $1,000 \mathrm{mg}$ versus $2,000 \mathrm{mg}$ in patients with chronic lymphocytic leukemia (CLL): results of the phase II GAGE (GAO4768g) trial. Poster presented at: American Society of Clinical Oncology Annual Meeting, 2014, Alexandria, VA.

31. Goede V, Fischer K, Busch R, et al. Obinutuzumab plus chlorambucil in patients with CLL and coexisting conditions. N Engl J Med. 2014; 370(12):1101-1110

32. Parmelee PA, Thuras PD, Katz IR, Lawton MP. Validation of the Cumulative Illness Rating Scale in a geriatric residential population. J Am Geriatr Soc. 1995;43(2):130-137.

33. Brown JR, O'Brien S, Kingsley D, Eradat H. Safety and efficacy of obinutuzumab (GA101) with fludarabine/cyclophosphamide (G-FC) or bendamustine (G-B) in the initial therapy of patients with chronic lymphocytic leukemia (CLL): results from the Phase 1b Galton Trial (GAO4779g). Poster presented at: American Society of Hematology Annual Meeting, 2013, Washington, DC.

34. Golay J, Semenzato G, Rambaldi A, et al. Lessons for the clinic from rituximab pharmacokinetics and pharmacodynamics. MAbs. 2013;5(6): 826-837.
OncoTargets and Therapy

\section{Publish your work in this journal}

OncoTargets and Therapy is an international, peer-reviewed, open access journal focusing on the pathological basis of all cancers, potential targets for therapy and treatment protocols employed to improve the management of cancer patients. The journal also focuses on the impact of management programs and new therapeutic agents and protocols on

\section{Dovepress}

patient perspectives such as quality of life, adherence and satisfaction. The manuscript management system is completely online and includes a very quick and fair peer-review system, which is all easy to use. Visit http://www.dovepress.com/testimonials.php to read real quotes from published authors. 